\title{
Role of isavuconazole in the treatment of invasive fungal infections
}

\author{
This article was published in the following Dove Press journal: \\ Therapeutics and Clinical Risk Management \\ 3 August 2016 \\ Number of times this article has been viewed
}

\author{
Dustin TWilson ${ }^{1,2}$ \\ $\checkmark$ Paul Dimondi 1,3 \\ Steven W Johnson ${ }^{1,4}$ \\ Travis M Jones' \\ Richard H Drew ${ }^{1,5}$ \\ 'Department of Pharmacy Practice, \\ Campbell University College of \\ Pharmacy \& Health Sciences, Buies \\ Creek, NC, USA; ${ }^{2}$ Department of \\ Pharmacy, Duke University Hospital, \\ Durham, NC, USA; ${ }^{3}$ Department of \\ Pharmacy, Durham VA Medical Center, \\ Durham, NC, USA; ${ }^{4}$ Department of \\ Pharmacy, Forsyth Medical Center, \\ Winston-Salem, NC, USA; ${ }^{5}$ Division of \\ Infectious Diseases, Duke University \\ Hospital, Durham, NC, USA
}

Correspondence: Dustin T Wilson Duke University Hospital, PO Box 3089, Durham, NC 277I0, USA

$\mathrm{Tel}+\mathrm{I} 9196846353$

$\mathrm{Fax}+19196812741$

Email dustin.wilson@duke.edu

\begin{abstract}
Despite recent advances in both diagnosis and prevention, the incidence of invasive fungal infections continues to rise. Available antifungal agents to treat invasive fungal infections include polyenes, triazoles, and echinocandins. Unfortunately, individual agents within each class may be limited by spectrum of activity, resistance, lack of oral formulations, significant adverse event profiles, substantial drug-drug interactions, and/or variable pharmacokinetic profiles. Isavuconazole, a second-generation triazole, was approved by the US Food and Drug Administration in March 2015 and the European Medicines Agency in July 2015 for the treatment of adults with invasive aspergillosis (IA) or mucormycosis. Similar to amphotericin B and posaconazole, isavuconazole exhibits a broad spectrum of in vitro activity against yeasts, dimorphic fungi, and molds. Isavuconazole is available in both oral and intravenous formulations, exhibits a favorable safety profile (notably the absence of QTc prolongation), and reduced drug-drug interactions (relative to voriconazole). Phase 3 studies have evaluated the efficacy of isavuconazole in the management of IA, mucormycosis, and invasive candidiasis. Based on the results of these studies, isavuconazole appears to be a viable treatment option for patients with IA as well as those patients with mucormycosis who are not able to tolerate or fail amphotericin B or posaconazole therapy. In contrast, evidence of isavuconazole for invasive candidiasis (relative to comparator agents such as echinocandins) is not as robust. Therefore, isavuconazole use for invasive candidiasis may initially be reserved as a step-down oral option in those patients who cannot receive other azoles due to tolerability or spectrum of activity limitations. Post-marketing surveillance of isavuconazole will be important to better understand the safety and efficacy of this agent, as well as to better define the need for isavuconazole serum concentration monitoring.
\end{abstract}

Keywords: isavuconazole, azole, antifungal, aspergillosis, Mucormycetes, mucormycosis

\section{Introduction}

Despite recent advances in both diagnosis and prevention, the incidence of invasive fungal infections (IFIs) continues to rise. ${ }^{1,2}$ The most common fungal pathogens associated with invasive disease in humans include Candida spp., Aspergillus spp., and Mucormycetes. ${ }^{3}$ Invasive candidiasis is the fourth most common health careassociated bloodstream infection in the US, and is associated with a $47 \%$ attributable mortality rate. ${ }^{4,5}$ Invasive aspergillosis (IA) is one of the most common life-threatening IFIs in immunocompromised patients, most notably in patients with hematologic malignancies or hematopoietic stem cell transplantations. ${ }^{6}$ The incidence of IA in hematopoietic stem cell transplantation patients has been reported as high as $15 \%$ with mortality rates ranging from $50 \%-90 \%$ at 1 year. ${ }^{2,6}$ Similar to IA, mucormycosis is most frequently diagnosed in patients with hematologic malignancies and those who received solid organ or hematologic stem cell transplantation. While not as prevalent 
as IA and invasive candidiasis (10-50-fold less frequent, respectively), the increasing number of breakthrough infections with Mucormycetes reported in patients receiving mold-active agents (eg, voriconazole and echinocandins) is concerning. ${ }^{7,8}$

Available antifungal agents to treat IFIs include polyenes, triazoles, and echinocandins. For years, amphotericin B (a polyene) was the treatment of choice for serious, lifethreatening IFIs. However, given its significant adverse event profile (most notably nephrotoxicity, infusion reactions, and electrolyte disturbances), its use has declined given the availability of safer alternatives in select infections. ${ }^{9}$ Despite these limitations, lipid-based formulations of amphotericin B have remained the treatment of choice for select IFIs. This includes mucormycosis, due (in part) to the lack of in vitro activity of most antifungals against these pathogens. ${ }^{7}$ Since the 1990 s, triazoles have provided an alternative to amphotericin B for treatment of many IFIs. Fluconazole represents a viable treatment option for many susceptible Candida spp.; however, widespread use as empiric therapy for all forms of invasive candidiasis has recently been limited by the emergence of azole-resistant spp. ${ }^{4}$ Voriconazole replaced amphotericin B as the preferred therapy for IA.${ }^{10}$ However, adverse events, drug-drug interactions, and both inter- and intra-patient pharmacokinetic variability (resulting in the need for serum concentration monitoring) complicate its use. ${ }^{9}$ Unlike other triazoles, posaconazole is often used in sequence after amphotericin B therapy in patients with mucormycosis or as primary therapy in patients unable to tolerate amphotericin B. ${ }^{7}$ Limited clinical experience with posaconazole in these patient populations, along with the caution against using the intravenous (IV) formulation in patients with a creatinine clearance $(\mathrm{CrCl})$ $<50 \mathrm{~mL} / \mathrm{min}$, have been barriers to its widespread use. The echinocandins (caspofungin, micafungin, and anidulafungin) were introduced in the mid-2000s, and have most often been used to treat invasive candidiasis and refractory IA. ${ }^{411}$ Despite widespread use of the echinocandins over the past decade, they are limited by their lack of oral formulations and absence of activity in vitro against Mucormycetes.

Isavuconazonium sulfate (Cresemba; Astellas Pharma US, Inc.; Northbrook, IL, USA) is a second-generation triazole approved by the US Food and Drug Administration (FDA) in March 2015 and the European Medicines Agency in July 2015. Isavuconazonium sulfate is the prodrug of isavuconazole (the active moiety), thus the latter terminology will be used throughout this review (except where noted). Isavuconazole exhibits a broad spectrum of activity in vitro against yeasts, dimorphic fungi, and molds (notably
Aspergillus spp. and Mucormycetes). ${ }^{12}$ Available in both oral and IV formulations, isavuconazole currently has approved indications for the treatment of adults with IA and mucormycosis. ${ }^{12}$ The primary aim of this review is to summarize the pharmacologic, kinetic, clinical efficacy and safety characteristics of isavuconazole which best help define its potential role in the management of IFIs.

\section{Pharmacology/spectrum of activity}

Similar to other azoles, isavuconazole inhibits the CYP450dependent enzyme lanosterol 14-alpha-demethylase, encoded by the gene ERG11. This enzyme is responsible for producing essential fungal cell wall components through conversion of lanosterol to ergosterol. ${ }^{12,13}$ As a result, fungal cell membrane fluidity and asymmetry are compromised. This leads to membrane dysfunction and arrested fungal growth.

Isavuconazole has demonstrated in vitro activity against a variety of fungal species including Candida, Cryptococcus, Aspergillus, and Mucormycetes (see Table 1). Among a variety of Candida spp., the minimum inhibitory concentration for $90 \%$ of isolates $\left(\mathrm{MIC}_{90}\right)$ generally ranges from $0.25-0.5 \mathrm{mcg} / \mathrm{mL}$ based on Clinical and Laboratory Standards Institute (CLSI) methodology. ${ }^{14,15}$ However, several species (including Candida glabrata and Candida krusei) displayed elevated MIC values against isavuconazole $(2 \mathrm{mcg} / \mathrm{mL}$ and $1 \mathrm{mcg} / \mathrm{mL}$, respectively). ${ }^{14,15}$ No apparent decline in in vitro activity was observed against Candida isolates over a 3-year period. ${ }^{15}$ Non-Candida yeasts are also generally susceptible to isavuconazole. For example, the $\mathrm{MIC}_{90}$ against a collection of Cryptococcus neoformans isolates was $0.12 \mathrm{mcg} / \mathrm{mL} .{ }^{14}$ When compared to the activity of voriconazole and posaconazole, the in vitro activity of isavuconazole is comparable against the majority of Candida spp. as well as non-Candida yeasts, and is more active than fluconazole. One notable exception is Candida guilliermondii where both voriconazole and posaconazole exhibited lower $\mathrm{MIC}_{90}$ values than isavuconazole ( $2 \mathrm{mcg} / \mathrm{mL}, 2 \mathrm{mcg} / \mathrm{mL}$ and $8 \mathrm{mcg} / \mathrm{mL}$, respectively). ${ }^{14}$

Isavuconazole is active in vitro against common Aspergillus spp., including Aspergillus fumigatus and Aspergillus flavus with an $\mathrm{MIC}_{90}$ of $1 \mathrm{mcg} / \mathrm{mL}$ for both organisms (using CLSI methods). ${ }^{14,16}$ Aspergillus niger exhibits reduced susceptibility to isavuconazole, with an $\mathrm{MIC}_{90}$ of $4 \mathrm{mcg} / \mathrm{mL}$. Overall, isavuconazole MICs for Aspergillus spp. are comparable to the activity of both voriconazole $(0.5 \mathrm{mcg} / \mathrm{mL})$ and posaconazole $(0.5 \mathrm{mcg} / \mathrm{mL})$.

Several studies have evaluated the activity of isavuconazole vs non-Aspergillus molds, specifically the Mucormycetes. A study which included Mucormycetes isolates $(n=72)$ 
Table I In vitro activity of isavuconazole against select pathogenic fungi

\begin{tabular}{|c|c|c|c|c|c|}
\hline \multirow[t]{2}{*}{ Organism } & \multirow[t]{2}{*}{ Isolates (n) } & \multicolumn{3}{|c|}{ Isavuconazole } & \multirow[t]{2}{*}{ References } \\
\hline & & $\mathrm{MIC}_{50} *$ & MIC $_{90} *$ & Range* & \\
\hline \multicolumn{6}{|l|}{ Candida spp. } \\
\hline C. albicans & 621 & 0.015 & 0.03 & $\leq 0.008$ to $>8$ & 14 \\
\hline C. glabrata & 235 & 0.5 & 2 & $0.03-8$ & 14 \\
\hline C. parapsilosis & 197 & 0.06 & 0.12 & $\leq 0.008-1$ & 14 \\
\hline C. tropicalis & 110 & 0.06 & 0.25 & $0.015-4$ & 14 \\
\hline C. krusei & 37 & 0.5 & 1 & $0.12-4$ & 14 \\
\hline C. lusitaniae & 33 & 0.03 & 0.06 & $\leq 0.008-0.12$ & 14 \\
\hline C. dubliniensis & 31 & 0.015 & 0.015 & $\leq 0.008-0.015$ & 14 \\
\hline \multicolumn{6}{|l|}{ Aspergillus spp. } \\
\hline A. fumigatus & 926 & 0.5 & I & 0.06 to $\geq 8$ & 14 \\
\hline A. flavus & 454 & 0.5 & 1 & $0.125-4$ & 14,16 \\
\hline A. niger & 218 & I & 2 & 0.06 to $>8$ & 14,16 \\
\hline A. terreus & 390 & 0.25 & 0.5 & $0.06-2$ & 14,16 \\
\hline A. nidulans & 106 & 0.125 & 1 & $0.06-1$ & 14,16 \\
\hline \multicolumn{6}{|l|}{ Other } \\
\hline Cryptococcus neoformans & 69 & 0.6 & 0.12 & $\leq 0.008-0.5$ & 14 \\
\hline Rhizomucor & 9 & 1 & - & $0.5-1$ & 17 \\
\hline Lichtheimia spp. & 17 & 1 & - & $0.5-2$ & 17 \\
\hline Rhizopus spp. & 32 & 1 & - & $0.5-4$ & 17 \\
\hline Mucor spp. & 13 & 4 & - & $1-8$ & 17 \\
\hline
\end{tabular}

Notes: *Concentration in $\mathrm{mcg} / \mathrm{mL} ;-$, data not reported.

Abbreviations: $\mathrm{n}$, number; $\mathrm{MIC}_{50}$, minimum inhibitory concentration for $50 \%$ of isolates; $\mathrm{MIC}_{90}$, minimum inhibitory concentration for $90 \%$ of isolates.

compared the in vitro activity of isavuconazole against amphotericin $\mathrm{B}$, voriconazole, and posaconazole. ${ }^{17}$ Using the European Committee on Antimicrobial Susceptibility Testing and CLSI testing methodologies, isavuconazole $\mathrm{MIC}_{50}$ ranged from 1-4 mcg/mL for isolates of Mucormycetes. However, some species (such as Mucor circinelloides) displayed reduced susceptibility, with the $\mathrm{MIC}_{50}$ ranging from $4-16 \mathrm{mcg} / \mathrm{mL}$. While isavuconazole exhibits higher $\mathrm{MIC}_{50}$ values than posaconazole $\left(\mathrm{MIC}_{50}\right.$ ranged from $0.25-1$ $\mathrm{mcg} / \mathrm{mL}$ ) against Mucormycetes isolates, the lack of defined breakpoints for these organisms makes the clinical significance of this finding uncertain.

\section{Resistance}

Resistance to triazole antifungals among Candida spp. continues to be a concern in clinical practice. ${ }^{13}$ The primary mechanisms for azole resistance in Candida spp. include over-expression of efflux pumps through $\mathrm{ABC}$ transporters, major facilitator transporter $M D R 1$, and changes in EGR11 gene encoding for 14-alpha-demethylase enzyme. ${ }^{13}$ In an in vitro study, the activity of isavuconazole was compared to fluconazole, itraconazole, posaconazole, and voriconazole among isolates of Saccharomyces cerevisiae, Candida albicans, and C. glabrata exhibiting a variety of azole resistance mechanisms. Over-expression of $\mathrm{ABC}$ transporters and mutations in ERG11 accounted for the loss of isavuconazole activity, while activity was not affected by mutations in $M D R 1 .{ }^{18}$ Since the efflux pumps encoded by MDRl are selective for fluconazole, isavuconazole could potentially be a useful agent for fluconazole-resistant Candida isolates with this mechanism. Unfortunately, Candida spp. can often possess multiple resistance genes, and it can be challenging in clinical practice to discern the type and number of resistance mechanisms with current susceptibility testing. While the most common mechanism cited for azole resistance in Aspergillus spp. is an alteration in the Cyp51A gene, recent reports have described many other potential areas of azole resistance in the absence of Cyp51A mutations. ${ }^{19}$ In vitro reports have demonstrated isavuconazole crossresistance in Aspergillus isolates with Cyp51A mutations resistant to other azole antifungals including itraconazole, posaconazole, and voriconazole. ${ }^{19}$

\section{Pharmacodynamics (animal model)}

The pharmacodynamics of isavuconazole have been evaluated in murine models of disseminated candidiasis and aspergillosis infections. ${ }^{20-23}$ In vivo murine invasive candidiasis models reported that the 24-hour free-drug area under the time-concentration curve (AUC) over the MIC was most predictive of isavuconazole efficacy $\left(R^{2}, 0.84\right) .{ }^{22}$ Of note was the observation that isavuconazole AUC/MIC values associated with efficacy were significantly lower for the $C$. glabrata 
and Candida tropicalis isolates compared to $C$. albicans $(P=0.04)$. A similar study evaluated the pharmacodynamic target of isavuconazole in IA. ${ }^{21}$ Fifty percent survival was attained with an AUC/MIC ratio of 24.73 (95\% confidence interval [CI], 22.50 to 27.18). As expected, isavuconazole was ineffective at improving survival in isolates harboring azole resistance genes and elevated MIC values.

\section{Pharmacokinetics}

Isavuconazole is commercially available as the prodrug isavuconazonium sulfate. ${ }^{12}$ The active moiety (isavuconazole) is rapidly produced through hydrolysis by plasma esterases. Animal model and human studies have described a high oral bioavailability of isavuconazole (98\%) (see Table 2). The oral absorption is not significantly affected by food, enabling isavuconazole to be taken without regard to meals. Single dose studies of IV (50, 100, or $200 \mathrm{mg})$ and oral $(100,200$, $400 \mathrm{mg}$ ) isavuconazole demonstrated peak plasma concentrations (Cmax) of $2-2.5 \mathrm{mcg} / \mathrm{mL}$ achieved at the end of the 1-hour infusion and 1.5 to 3 hours after oral administration. ${ }^{24}$ While disproportionate increases in plasma AUC and Cmax concentrations raised the possibility of non-linear drug exposure and saturable metabolism, this was not observed in subsequent pharmacokinetic studies. ${ }^{24,25}$ In multidose studies, isavuconazole steady-state concentrations were not reached until approximately 2 weeks of once-daily dosing. There was significant (3-5-fold) drug accumulation from study day 1 to study day 14 . However, the Cmax and AUC values were proportional for the $50 \mathrm{mg}$ and $100 \mathrm{mg}$ daily doses (indicating that isavuconazole follows nonsaturable linear elimination). ${ }^{25}$ Isavuconazole is extensively protein bound (99\%) similar to posaconazole and widely distributed (volume of distribution 155-494 L) which is consistent with other azole antifungals. ${ }^{24}$ Isavuconazole primarily undergoes hepatic metabolism through CYP3A4 and subsequently UGTs, with only $0.02 \%-0.38 \%$ of unchanged drug being recovered in urine. ${ }^{12}$ Elimination is biphasic, with a half-life of 0.42-2 hours and 56-104 hours in the distribution and terminal elimination phases, respectively. ${ }^{24}$ The prolonged half-life of isavuconazole compared to other azole antifungals allows for once-daily maintenance dosing.

The pharmacokinetic profile of isavuconazole has been reported in special populations, including hepatic impairment, renal impairment, and geriatrics. The increased water solubility of the isavuconazole prodrug (isavuconazonium sulfate) facilitates formation of a solution without addition of a cyclodextrin which limits the use of IV voriconazole and posaconazole due to the risk of renal toxicity in patients with a $\mathrm{CrCl}<50 \mathrm{~mL} / \mathrm{min}$. Due to the lack of cyclodextrin and minimal renal excretion, isavuconazole can be given to patients with mild, moderate, and severe renal impairment without need for dose adjustment. Patients with mild to moderate hepatic impairment (Child-Pugh Class A or B) exhibit decreases in clearance ( $40 \%$ and $48 \%$, respectively), but such reductions do not warrant dose reduction in these patients. ${ }^{26,27}$ Currently, there are no data in patients with severe hepatic impairment (Child-Pugh Class C). ${ }^{12}$ No published data exist which report the pharmacokinetics of isavuconazole in pediatric patients. AUC does not change significantly in elderly males ( $>65$ years of age); however there are slight increases for elderly females when compared to elderly males and younger females (AUC increase 38\% and 47\%, respectively). Nevertheless, these increases were not considered significant enough to warrant dose modification. ${ }^{12}$ Finally, the pharmacokinetics of isavuconazole were assessed in a pharmacokinetic model between healthy Western and Chinese subjects. ${ }^{12}$

Table 2 Comparison of pharmacokinetic parameters of selected triazoles

\begin{tabular}{|c|c|c|c|c|}
\hline Parameter & Fluconazole ${ }^{48}$ & Voriconazole 49,50 & Posaconazole ${ }^{50,51}$ & Isavuconazole ${ }^{12,50}$ \\
\hline Available & Oral tablets & Oral tablets & Oral suspension & Oral capsules \\
\hline \multirow[t]{2}{*}{ Formulations } & Oral suspension & Oral suspension & Oral tablets & Intravenous \\
\hline & Intravenous & Intravenous & Intravenous & \\
\hline Oral bioavailability, \% & $>90$ & $>90^{\mathrm{a}}$ & Range $8-47^{b}$ & 98 \\
\hline Volume of distribution, (L/kg) & 0.6 & 4.6 & Range 5-25 & Range 4.4-7.7 \\
\hline Protein binding, $\%$ & Range $11-12$ & 58 & $>99$ & 99 \\
\hline CSF penetration (relative) & Good & Good & Poor & Poor \\
\hline Urine concentration - active drug & Good & Poor & Poor & Poor \\
\hline Metabolism (primary route) & $\begin{array}{l}\text { Minor hepatic (predominately } \\
\text { renally eliminated) }\end{array}$ & Hepatic & Hepatic & Hepatic \\
\hline Elimination (primary route) & Renal & Renal & Fecal & Fecal \\
\hline Half-life, hours & 31 & 6 & Range 25-35 & Range 56-104 \\
\hline
\end{tabular}

Notes: aDecreased when administered with food; 'oral tablets have higher bioavailability than oral suspension. Abbreviation: CSF, cerebrospinal fluid. 
Compared to Western subjects, Chinese subjects had a $40 \%$ lower clearance of isavuconazole. Despite the reduction in clearance, there is no formal dose adjustments for Chinese patients.

\section{Clinical studies Candidiasis}

The safety and efficacy of oral isavuconazole for the treatment of uncomplicated esophageal candidiasis was evaluated in a Phase 2, randomized, double-blind, parallel group, noninferiority study at eight sites in South Africa. ${ }^{28}$ Adults $18-65$ years of age were randomized (1:1:1:1) to receive one of the following once-daily oral regimens for a minimum of 14 days: isavuconazole $200 \mathrm{mg}$ on day 1 followed by $50 \mathrm{mg}$ from day 2 to the end-of-therapy (EOT); isavuconazole $400 \mathrm{mg}$ on days $1,7,14$, and 21 ; isavuconazole $400 \mathrm{mg}$ on day 1 , followed by $100 \mathrm{mg}$ from day 2 to the EOT; or fluconazole $200 \mathrm{mg}$ on day 1, followed by 100 $\mathrm{mg}$ from day 2 to the EOT. Approximately one third of study patients had HIV infection. C. albicans was isolated in $96.6 \%$ of cases, followed by $C$. glabrata $(2.8 \%)$ and C. tropicalis $(0.7 \%)$. Endoscopically-confirmed clinical response at the EOT was observed in $146(95.4 \%)$ patients, and was similar between treatment groups. Overall, each dosing regimen of isavuconazole demonstrated noninferiority (within a margin of $15 \%$ ) to fluconazole. Of note, the dose of fluconazole used in this study is lower than the dose currently recommended by the Infectious Diseases Society of America for this indication. ${ }^{4}$ In addition, patients with recurrent or refractory disease and those having failed prior antifungal therapy were excluded from this trial, which represent a large portion of patients in need of well-tolerated alternatives with oral dosage formulations.

The ACTIVE trial was a Phase 3, randomized, doubleblind, parallel-group study evaluating the safety and efficacy of isavuconazole (200 mg IV every 8 hours for six doses, followed by $200 \mathrm{mg}$ IV daily) compared to caspofungin $70 \mathrm{mg}$ IV daily in the treatment of candidemia and invasive candidiasis. ${ }^{29}$ After day 10, isavuconazole-treated patients had the option to step down to oral isavuconazole $200 \mathrm{mg}$ daily, and caspofungin-treated patients had the option to step down to oral voriconazole 400 mg every 12 hours until EOT (maximum of 56 days). Overall treatment response at the end of IV therapy, as assessed by the Data Review Committee, occurred in $60.3 \%$ and $71.1 \%$ of patients treated with isavuconazole and caspofungin, respectively (adjusted treatment difference of $-10.8 \% ; 95 \% \mathrm{CI},-19.9$ to $-1.8 \%) .{ }^{30}$ The study failed to meet criteria for noninferiority of isavuconazole in overall treatment response at the end of IV therapy. These results are consistent with another published study indicating superiority of anidulafungin over fluconazole for primary therapy in patients with invasive candidiasis. ${ }^{31}$

\section{Aspergillosis}

Isavuconazole has been FDA-approved for the treatment of IA based on results from the SECURE study, a Phase 3, randomized, double-blind, multicenter, active-controlled, non-inferiority study. ${ }^{32}$ This study evaluated the safety and efficacy of isavuconazole vs voriconazole for the primary treatment of IFIs in 516 patients. Adults with proven, probable, or possible IFIs (as defined by the European Organization for Research and Treatment of Cancer/ Mycoses Study Group criteria) caused by Aspergillus spp. or other filamentous fungi were randomized 1:1 to receive isavuconazole (200 mg IV every 8 hours for six doses followed by $200 \mathrm{mg}$ IV or oral daily thereafter) or voriconazole $(6 \mathrm{mg} / \mathrm{kg}$ IV every 12 hours for two doses followed by $4 \mathrm{mg} / \mathrm{kg}$ IV every 12 hours or $200 \mathrm{mg}$ orally every 12 hours thereafter). Included patients received treatment for at least 7 days following resolution of clinical signs or symptoms, up to a maximum duration of 84 days. Patients with moderate to severe renal dysfunction (defined as a calculated $\mathrm{CrCl}<50 \mathrm{~mL} / \mathrm{min}$ ) and patients treated with systemic mold-active antifungals for at least 4 days within the 7 days prior to receipt of first dose of study drug were excluded. The primary outcome was all-cause mortality through day 42 in the intention-to-treat population with a noninferiority margin of $10 \%$. More than $80 \%$ of patients in each group had an underlying hematological malignancy at baseline, and 63\% (isavuconazole) and 68\% (voriconazole) were neutropenic. Of note, $36 \%$ of patients in the isavuconazole group and $35 \%$ of patients in the voriconazole group were diagnosed by serum galactomannan assay. The allcause mortality of $18.6 \%$ and $20.2 \%$ in isavuconazole and voriconazole-treated patients (respectively) met criteria for noninferiority (adjusted treatment difference $-1.0 \%$; 95\% CI, -7.8 to 5.7). All-cause mortality through day 84 was $29 \%$ and $31 \%$, respectively. For the subset of patients with proven or probable IA $(\mathrm{n}=231)$, both all-cause mortality $(18.7 \%$ and $22.2 \%$, respectively) and overall clinical, mycological, and radiologic response at EOT (35\% and $38.9 \%$, respectively) were comparable. This study has important limitations worth discussing. First, patients undergoing treatment for hematological malignancies were not eligible for inclusion if they were receiving mold-active prophylaxis, which is common clinical practice. Thus, the efficacy of isavuconazole for 
breakthrough infections remains uncertain. ${ }^{33}$ Additionally, Aspergillus spp. was the most common causative pathogen for patients with culture-confirmed IFIs, and most patients were treated for lower respiratory tract disease, which limits these data in the generalizability of isavuconazole treatment for other molds or for extrapulmonary infections. ${ }^{33}$ Third, over one third of patients in each group were diagnosed with serum galactomannan testing. Finally, serum concentration monitoring of voriconazole was not allowed during the study. Studies have linked voriconazole serum concentrations to improved treatment responses in patients with IFIs. ${ }^{34}$

\section{Mucormycosis}

The safety and efficacy of isavuconazole for primary or salvage treatment of IA in patients with renal impairment and for rare IFIs, including mucormycosis was evaluated in a Phase 3, single-arm, open-label, multicenter, noncomparative trial. ${ }^{35}$ Adult patients with renal impairment and proven, probable, or possible IA were included. Additionally, patients with culture-confirmed IFIs caused by rare molds, including Mucormycetes were eligible. Included patients received isavuconazole $200 \mathrm{mg}$ IV or orally every 8 hours for six doses, followed by $200 \mathrm{mg}$ daily for up to 180 days or longer in patients with clinical benefit. Of the 149 patients enrolled, 37 had proven $(86 \%)$ or probable $(14 \%)$ mucormycosis only; the majority of which were lower respiratory tract infections (59.4\%) with or without other organ involvement. Pathogens causing mucormycosis included Mucorales not otherwise specified (35\%), Rhizopus oryzae (19\%), Mucor spp. (16\%), and Rhizomucor spp. (14\%). The median duration of treatment was 84 days (interquartile range 19-179, range 2-882). Overall, eleven (31.4\%) patients had a successful response at EOT. All-cause mortality at days 42 and 84 were $38 \%$ and $43 \%$, respectively, which is similar to published data on amphotericin B and posaconazole oral solution in immunocompromised hosts. ${ }^{36-38}$ Patients receiving isavuconazole for primary treatment had a lower mortality through day 42 compared with those receiving salvage therapy for refractory disease or those intolerant to other antifungals $(33.3 \%$, $45.5 \%$, and $40 \%$, respectively).

To further define the clinical efficacy of isavuconazole, a case-control analysis was reported in which patients with mucormycosis who received primary treatment with isavuconazole were compared to contemporary controls from the FungiScope registry who received amphotericin B followed by posaconazole. ${ }^{35}$ The crude (33\% vs $39 \%, P=0.775$ ) and weighted all-cause mortality at day $42(33 \%$ vs $41 \%$, $P=0.595)$ and survival at day $84(57 \%$ vs $50 \%, P=0.653)$ did not differ for patients treated with either isavuconazole or amphotericin B formulations, respectively.

\section{Safety and tolerability}

Clinically significant or commonly associated adverse effects with triazoles include gastrointestinal (nausea, vomiting, hepatic enzyme elevations) and cardiovascular (QTc prolongation) effects. ${ }^{9}$ However, the severity and incidence to which these occur vary between the different triazoles. In general, isavuconazole is well-tolerated and appears to have a similar safety profile to that of fluconazole. ${ }^{39}$ In Phase 1 and 2 studies, no serious adverse effects occurred due to isavuconazole. ${ }^{24,25,28}$ Furthermore, in Phase 3 clinical trials, isavuconazole appears to have a more favorable safety profile compared to that of voriconazole and a comparable safety profile to that of caspofungin. ${ }^{30,32,35}$ The most frequently reported adverse events were gastrointestinal disorders (nausea [26\%], vomiting [25\%], diarrhea [22\%], constipation [13\%], elevated liver enzymes [16\%]), headache (17\%), hypokalemia (14\%), dyspnea (12\%), cough (12\%), peripheral edema (11\%), and back pain (10\%). In the SECURE study, isavuconazole-treated patients had significantly fewer hepatobiliary disorders ( $9 \%$ vs $16 \% ; P=0.016)$, skin and subcutaneous tissue disorders (33\% vs $42 \% ; P=0.037)$, and eye disorders $(15 \%$ vs $27 \% ; P=0.002)$ compared to voriconazole-treated patients. ${ }^{32}$ In addition, significantly fewer patients reported treatment-emergent adverse events considered drug-related by investigators with isavuconazole compared to voriconazole ( $42 \%$ vs $60 \% ; P<0.001)$. Drug discontinuation due to either treatment-emergent or drugrelated adverse events was less common with isavuconazole compared to voriconazole ( $14 \%$ vs $23 \%$ and $8 \%$ vs $14 \%$, respectively). ${ }^{32}$ However, the increased number of adverse events and drug discontinuations in the voriconazole group may be attributable (at least in part) to the inability to monitor voriconazole serum concentrations. The frequency of treatment-emergent (94.6\%) and study-drug related (35.1\%) adverse events in patients treated for invasive mucormycosis in the VITAL study were similar to the SECURE study. ${ }^{35}$ The most common treatment-emergent adverse events were nausea, vomiting, diarrhea, and pyrexia.

Cardiovascular adverse events (most notably QTc prolongation) are a significant concern with the available triazoles. This concern may be enhanced with drug interactions that affect the metabolism of many QT-prolonging medications. ${ }^{40}$ However, unlike current triazoles, most studies have not shown an association with isavuconazole and QTc prolongation with the exception of the VITAL study. ${ }^{25,26,35}$ 
In the VITAL study, $25 \%$ of patients had an increase in QTc of more than $30 \mathrm{msec}^{35}$ This finding will need to be evaluated in future studies to better delineate its potential clinical significance. On the other hand, isavuconazole was noted to cause a dose- and concentration-related QTc interval shortening in other studies. When isavuconazole is administered at the maintenance dose of $200 \mathrm{mg}$ daily, the least squares mean difference in the QTc from placebo was $-13.1 \mathrm{msec}$ (90\% CI: -17.1, -9.1). ${ }^{12}$ In clinical studies, all occurrences of QTc shortening were temporary and did not require intervention. The clinical significance of isavuconazoleinduced QTc shortening is unknown; however, patients with familial short-QT syndrome should not receive therapy with isavuconazole..$^{12,41,42}$ Furthermore, caution is warranted during the coadministration of isavuconazole with medications known to shorten the QT interval (ie, lamotrigine, primidone, rufinamide)..$^{12,41,42}$

\section{Drug interactions}

The triazoles have a significant number of drug-drug interactions, especially with itraconazole and voriconazole. ${ }^{9}$ These interactions are facilitated primarily through their variable inhibition of the cytochrome P450 system (most notably CYP3A4). ${ }^{43}$ Isavuconazole's interaction profile most closely resembles that of posaconazole, and appears to have less drug interactions than voriconazole. ${ }^{39,40}$ Specifically, in vitro studies have shown isavuconazole to be a substrate of both CYP3A4 and CYP3A5, inhibitor of CYP3A4, CYP2C8, CYP2C9, CYP2C19, CYP2D6, P-gp, BCRP, and human OCT2. Isavuconazole is also a weak inducer of CYP3A4, CYP2B6, CYP2C8, and CYP2C9. ${ }^{12}$ However, in vivo drug-drug interaction studies have demonstrated isavuconazole to be a mild-moderate inhibitor of CYP3A4, a mild inducer of CYP2B6, a mild inhibitor of P-gp and UGT, and did not affect the pharmacokinetics of substrates of CYP1A2, CYP2C8, CYP2C9, CYP2C19, or CYP2D6 (see Table 3). ${ }^{12}$ Consequently, isavuconazole is contraindicated with concomitant administration of strong inducers

Table 3 Comparative inhibition of selected CYP450 isoenzymes by triazoles

\begin{tabular}{lllll}
\hline Azole & CYP2C8 & CYP2C9 & CYP2C19 & CYP3A4 \\
\hline Fluconazole & ++ & ++ & + & ++ \\
Itraconazole & + & + & - & +++ \\
Voriconazole & ++ & ++ & +++ & ++ \\
Posaconazole & - & - & - & +++ \\
Isavuconazole & - & - & - & $+1++$ \\
\hline
\end{tabular}

Notes: -, no inhibition; +, mild inhibition; ++, moderate inhibition; +++, strong inhibition. Data from references. ${ }^{12,52,53}$ and inhibitors of CYP3A4 such as ketoconazole, rifampin, carbamazepine, and St John's wort (see Table 4). Similar to other triazoles, concomitant administration of isavuconazole with immunosuppressive agents (cyclosporine, tacrolimus, and sirolimus) requires potential dose reduction and more frequent monitoring of the immunosuppressive agents. Currently, there are no formal dose reduction recommendations. However, authors of a recent case report recommend an initial 50\% reduction in tacrolimus dose when used in combination with isavuconazole. ${ }^{44}$ Furthermore, medications that have a narrow therapeutic window that are substrates of $\mathrm{P}-\mathrm{gp}$ (eg, digoxin) require therapeutic drug monitoring (TDM) and possible dosage adjustments. ${ }^{12}$ Of note, isavuconazole is not affected by medications which alter the gastric $\mathrm{pH}$, unlike itraconazole and the suspension formulation of posaconazole.

\section{Dosing and administration}

Isavuconazole is administered orally and intravenously as the prodrug isavuconazonium sulfate. Isavuconazole capsules contain $186 \mathrm{mg}$ of isavuconazonium sulfate, which is equivalent to $100 \mathrm{mg}$ of isavuconazole. ${ }^{12}$ The capsules can be administered without regard to food, unlike voriconazole and the oral formulations of posaconazole. ${ }^{9}$ Isavuconazole injection is supplied as a single-dose vial containing $372 \mathrm{mg}$ of isavuconazonium sulfate (equivalent to $200 \mathrm{mg}$ of isavuconazole). The reconstituted injection should be added to a compatible diluent ( $0.9 \%$ sodium chloride or $5 \%$ dextrose) and administered through an in-line filter over at least 1 hour. ${ }^{12}$

The recommended dosing of isavuconazole is to give a loading dose of $372 \mathrm{mg}$ isavuconazonium sulfate $(200 \mathrm{mg}$ of isavuconazole) every 8 hours for six doses, followed by a maintenance dose of $372 \mathrm{mg}$ isavuconazonium sulfate (200 mg of isavuconazole) once daily. ${ }^{12}$ The maintenance dose can be started 12-24 hours after the last loading dose. As discussed previously, dosage adjustments are not warranted in patients with renal disease, mild to moderate liver disease, or advanced age. Isavuconazole has not adequately been studied in patients $<18$ years of age, thus its use should be avoided in these patients due to lack of available data. In addition, animal studies have shown that isavuconazole is excreted in breast milk; therefore, nursing mothers should not receive therapy with this agent. Lastly, isavuconazole should only be utilized in pregnant patients or patients with severe hepatic impairment (Child-Pugh Class C) if the potential benefits outweigh the risks associated with therapy. ${ }^{12}$ 
Table 4 Selected drug-drug interactions with isavuconazole

\begin{tabular}{|c|c|}
\hline Medication & Clinical significance \\
\hline Ketoconazole & $\begin{array}{l}\text { - Isavuconazole's AUC increases by } \sim 5 \text {-fold when administered with concomitant ketoconazole } \\
\text { - Use with strong CYP3A4 inhibitors is contraindicated }\end{array}$ \\
\hline Rifampin & $\begin{array}{l}\text { - Isavuconazole's AUC decreases by } \sim 2 \text {-fold with concomitant rifampin } \\
\text { - Use with strong CYP3A4 inducers is contraindicated }\end{array}$ \\
\hline Lopinavir/ritonavir & $\begin{array}{l}\text { - Isavuconazole's AUC increases by } \sim 2 \text {-fold with concomitant lopinavir/ritonavir } \\
\text { - Use with caution }\end{array}$ \\
\hline $\begin{array}{l}\text { Sirolimus, tacrolimus, cyclosporine, } \\
\text { and mycophenolate mofetil }\end{array}$ & $\begin{array}{l}\text { - Isavuconazole increases the AUC of sirolimus and tacrolimus by } \sim 2 \text {-fold, cyclosporine by } \sim 1.25 \text {-fold, } \\
\text { and mycophenolate mofetil by } \sim 1.5 \text {-fold with concomitant administration } \\
\text { - Use with caution, TDM of sirolimus, tacrolimus, and cyclosporine is recommended } \\
\text { - } 50 \% \text { dose reduction of tacrolimus has been recommended }\end{array}$ \\
\hline Atorvastatin, and digoxin & $\begin{array}{l}\text { - Isavuconazole increases the AUC of these agents by } \sim 1.25 \text {-fold with concomitant administration } \\
\text { - Use with caution, TDM should be performed with digoxin }\end{array}$ \\
\hline Midazolam & $\begin{array}{l}\text { - Isavuconazole increases the AUC of midazolam by } \sim 2 \text {-fold with concomitant administration } \\
\text { - Use with caution }\end{array}$ \\
\hline
\end{tabular}

Note: Data from references. ${ }^{2,44}$

Abbreviations: AUC, area under the time-concentration curve; TDM, therapeutic drug monitoring.

\section{Serum concentration monitoring}

Due to significant inter-patient and intra-patient variability in serum concentrations, serum concentration monitoring is often utilized for most triazoles (itraconazole, voriconazole, and posaconazole) for select indications. ${ }^{9,34,45}$ Similar to fluconazole, routine serum concentration monitoring is not currently required with isavuconazole. However, pharmacokinetic studies have shown that Chinese patients have a $40 \%$ lower clearance of isavuconazole compared to Western patients suggesting that CYP polymorphisms may play an important role in the metabolism of the drug. ${ }^{12}$ In addition, isavuconazole is a substrate of CYP3A4, thus inhibitors and inducers of this isoenzyme can affect the serum concentrations of isavuconazole. Considering the possibility of inter- and intra-patient variability with isavuconazole, case reports and clinical trials have reported TDM with isavuconazole. . $^{32,35,44,46,47}$ However, correlation between serum concentrations and adverse effects and efficacy have not been fully assessed at this time. ${ }^{40}$ Since the need for isavuconazole TDM remains unclear, further investigation is warranted.

\section{Potential place in therapy for IFIs}

Features of isavuconazole include a broad spectrum of antifungal activity, availability of both oral and IV formulations, favorable safety profile (notably the absence of QTc prolongation), reduced drug-drug interactions (relative to voriconazole), and favorable pharmacokinetic profile. To date, isavuconazole has been evaluated for treatment of IA, mucormycosis, and candidiasis.

Isavuconazole has been shown to be non-inferior to voriconazole in patients with IA. ${ }^{32}$ In addition, isavuconazole was associated with fewer adverse events and drug discontinuations. Therefore, isavuconazole can be used as alternative therapy in patients with IA who cannot tolerate voriconazole therapy due to severe adverse effects (hepatotoxicity, skin reactions, and visual disturbances) or have prolonged QTc prior to therapy or while on voriconazole therapy. Isavuconazole may also be utilized in those patients with IA who have $\mathrm{CrCl}<50 \mathrm{~mL} / \mathrm{min}$ and require IV therapy since its formulation does not contain cyclodextrin.

Currently, the mainstays of therapies for mucormycosis are amphotericin B formulations and posaconazole. Amphotericin B is often utilized as primary therapy for mucormycosis, while posaconazole is often used in sequence after amphotericin B therapy or as primary therapy in those patients unable to tolerate amphotericin B therapy. Isavuconazole is as effective as current primary and salvage therapy for patients with mucormycosis even though the evidence comes from a small number of patients $(n=37) .{ }^{35}$ Outcomes are similar to those of amphotericin B formulations when used as primary therapy. Considering the significant adverse effects of amphotericin B formulations (most notably nephrotoxicity), the limited evidence to support posaconazole as primary therapy and the inability to use IV posaconazole in patients with reduced renal function, isavuconazole may be an invaluable option for the primary therapy of mucormycosis. In addition, isavuconazole can serve as an alternative to oral formulations of posaconazole for sequence therapy.

Finally, the current evidence of isavuconazole in other IFIs, notably candidiasis, is not as robust as compared to IA and mucormycosis. The ACTIVE study did not show noninferiority of isavuconazole to caspofungin in patients with candidemia and invasive candidiasis. ${ }^{30}$ Based on these data, 
it appears that echinocandins will remain as preferred initial therapy for invasive candidiasis. However, isavuconazole may be a reasonable step-down oral option in those patients who cannot receive other azoles due to tolerability or spectrum of activity limitations.

\section{Conclusion}

IFIs, notably IA and mucormycosis, are associated with significant morbidity and mortality, especially in immunocompromised patients. Current treatment options for these dreaded infections are plagued with shortcomings. Isavuconazole is a broad-spectrum triazole with a good pharmacokinetic profile and favorable safety and drug-drug interaction profiles. Based on the current data, isavuconazole appears to be a viable alternative treatment option for patients with IA who are intolerant of voriconazole as well as those patients with mucormycosis who are not able to tolerate or fail amphotericin B or posaconazole therapy. Given the lack of "real world" clinical experience with isavuconazole, postmarketing surveillance will be important to understand the true degree of safety of this agent as well as the role of serum concentration monitoring.

\section{Disclosure}

The authors report no conflicts of interest relevant to this work.

\section{References}

1. Carrillo-Munoz AJ, Finquelievich J, Tur-Tur C, et al. Combination antifungal therapy: a strategy for the management of invasive fungal infections. Rev Esp Quimioter. 2014;27(3):141-158.

2. Kousha M, Tadi R, Soubani AO. Pulmonary aspergillosis: a clinical review. Eur Respir Rev. 2011;20(121):156-174.

3. Koehler P, Cornely OA. Contemporary Strategies in the Prevention and Management of Fungal Infections. Infect Dis Clin North Am. 2016; 30(1):265-275.

4. Pappas PG, Kauffman CA, Andes DR, et al. Clinical Practice Guideline for the Management of Candidiasis: 2016 Update by the Infectious Diseases Society of America. Clin Infect Dis. 2016;62(4):e1-e50.

5. Antibiotic Resistance Threats in the United States, 2013. Centers for Disease Control and Prevention; 2014. Available from: http://www. cdc.gov/drugresistance/threat-report-2013/. Accessed April 22, 2016.

6. Leeflang MM, Debets-Ossenkopp YJ, Wang J, et al. Galactomannan detection for invasive aspergillosis in immunocompromised patients. Cochrane Database Syst Rev. 2015;12:CD007394.

7. Farmakiotis D, Kontoyiannis DP. Mucormycoses. Infect Dis Clin North Am. 2016;30(1):143-163.

8. Petrikkos G, Skiada A, Lortholary O, Roilides E, Walsh TJ, Kontoyiannis DP. Epidemiology and clinical manifestations of mucormycosis. Clin Infect Dis. 2012;54 Suppl 1:S23-S34.

9. Allen D, Wilson D, Drew R, Perfect J. Azole antifungals: 35 years of invasive fungal infection management. Expert Rev Anti Infect Ther. 2015;13(6):787-798.

10. Walsh TJ, Anaissie EJ, Denning DW, et al. Treatment of aspergillosis: clinical practice guidelines of the Infectious Diseases Society of America. Clin Infect Dis. 2008;46(3):327-360.
11. Marr KA, Schlamm HT, Herbrecht R, et al. Combination antifungal therapy for invasive aspergillosis: a randomized trial. Ann Intern Med. 2015;162(2):81-89.

12. Cresemba ${ }^{\circledR}$ (isavuconazonium sulfate) [package insert]. Northbrook, IL: Astellas Pharma US, Inc.; 2015.

13. Pfaller MA. Antifungal drug resistance: mechanisms, epidemiology, and consequences for treatment. Am J Med. 2012;125(1 Suppl):S3-S13.

14. Pfaller MA, Rhomberg PR, Messer SA, Jones RN, Castanheira M. Isavuconazole, micafungin, and 8 comparator antifungal agents' susceptibility profiles for common and uncommon opportunistic fungi collected in 2013: temporal analysis of antifungal drug resistance using CLSI species-specific clinical breakpoints and proposed epidemiological cutoff values. Diagn Microbiol Infect Dis. 2015;82(4):303-313.

15. Pfaller MA, Messer SA, Rhomberg PR, Jones RN, Castanheira M. In vitro activities of isavuconazole and comparator antifungal agents tested against a global collection of opportunistic yeasts and molds. J Clin Microbiol. 2013;51(8):2608-2616.

16. Espinel-Ingroff A, Chowdhary A, Gonzalez GM, et al. Multicenter study of isavuconazole MIC distributions and epidemiological cutoff values for Aspergillus spp. for the CLSI M38-A2 broth microdilution method. Antimicrob Agents Chemother. 2013;57(8):3823-3828.

17. Arendrup MC, Jensen RH, Meletiadis J. In Vitro Activity of Isavuconazole and Comparators against Clinical Isolates of the Mucorales Order. Antimicrob Agents Chemother. 2015;59(12):7735-7742.

18. Sanglard D, Coste AT. Activity of Isavuconazole and Other Azoles against Candida Clinical Isolates and Yeast Model Systems with Known Azole Resistance Mechanisms. Antimicrob Agents Chemother. 2015; 60(1):229-238.

19. Chowdhary A, Kathuria S, Randhawa HS, Gaur SN, Klaassen CH, Meis JF. Isolation of multiple-triazole-resistant Aspergillus fumigatus strains carrying the TR/L98H mutations in the cyp51A gene in India. J Antimicrob Chemother. 2012;67(2):362-366.

20. Warn PA, Sharp A, Parmar A, Majithiya J, Denning DW, Hope WW. Pharmacokinetics and pharmacodynamics of a novel triazole, isavuconazole: mathematical modeling, importance of tissue concentrations, and impact of immune status on antifungal effect. Antimicrob Agents Chemother. 2009;53(8):3453-3461.

21. Seyedmousavi S, Bruggemann RJ, Meis JF, Melchers WJ, Verweij PE, Mouton JW. Pharmacodynamics of isavuconazole in an Aspergillus fumigatus mouse infection model. Antimicrob Agents Chemother. 2015;59(5):2855-2866.

22. Lepak AJ, Marchillo K, VanHecker J, Diekema D, Andes DR. Isavuconazole pharmacodynamic target determination for Candida species in an in vivo murine disseminated candidiasis model. Antimicrob Agents Chemother. 2013;57(11):5642-5648.

23. Lepak AJ, Marchillo K, Vanhecker J, Andes DR. Isavuconazole (BAL4815) pharmacodynamic target determination in an in vivo murine model of invasive pulmonary aspergillosis against wild-type and cyp51 mutant isolates of Aspergillus fumigatus. Antimicrob Agents Chemother. 2013;57(12):6284-6289.

24. Schmitt-Hoffmann A, Roos B, Heep M, et al. Single-ascending-dose pharmacokinetics and safety of the novel broad-spectrum antifungal triazole BAL4815 after intravenous infusions (50, 100, and 200 milligrams) and oral administrations (100, 200, and 400 milligrams) of its prodrug, BAL8557, in healthy volunteers. Antimicrob Agents Chemother. 2006;50(1):279-285.

25. Schmitt-Hoffmann A, Roos B, Maares J, et al. Multiple-dose pharmacokinetics and safety of the new antifungal triazole BAL4815 after intravenous infusion and oral administration of its prodrug, BAL8557, in healthy volunteers. Antimicrob Agents Chemother. 2006;50(1):286-293.

26. Schmitt-Hoffmann A, Roos B, Spickermann J, et al. Effect of mild and moderate liver disease on the pharmacokinetics of isavuconazole after intravenous and oral administration of a single dose of the prodrug BAL8557. Antimicrob Agents Chemother. 2009;53(11):4885-4890.

27. Desai A, Schmitt-Hoffmann AH, Mujais S, Townsend R. Population Pharmacokinetics of Isavuconazole in Subjects with Mild and Moderate Hepatic Impairment. Antimicrob Agents Chemother. 2016;60(5): 3025-3031. 
28. Viljoen J, Azie N, Schmitt-Hoffmann AH, Ghannoum M. A phase 2, randomized, double-blind, multicenter trial to evaluate the safety and efficacy of three dosing regimens of isavuconazole compared with fluconazole in patients with uncomplicated esophageal candidiasis. Antimicrob Agents Chemother. 2015;59(3):1671-1679.

29. Isavuconazole (BAL8557) in the Treatment of Candidemia and Other Invasive Candida Infections. Astellas Pharma Inc. Available from: https:/clinicaltrials.gov/ct2/show/NCT00413218?term=NCT0041321 8\&rank=1. NLM identifier: NCT00413218. Accessed June 21, 2016.

30. Astellas Pharma US, Inc. Astellas provides update on phase 3 study evaluating isavuconazole in patients with candidemia and other invasive candidal infections [news release]. Northbrook, IL: Astellas Pharma US, Inc.; 2015 [July 30]. Available from: http://newsroom. astellas.us/2015-07-30-Astellas-Provides-Update-on-Phase-3-StudyEvaluating-Isavuconazole-in-Patients-with-Candidemia-and-OtherInvasive-Candida-Infections. Accessed March 13, 2016.

31. Reboli AC, Rotstein C, Pappas PG, et al. Anidulafungin versus fluconazole for invasive candidiasis. N Engl J Med. 2007;356(24):2472-2482.

32. Maertens JA, Raad II, Marr KA, et al. Isavuconazole versus voriconazole for primary treatment of invasive mould disease caused by Aspergillus and other filamentous fungi (SECURE): a phase 3, randomisedcontrolled, non-inferiority trial. Lancet. 2016;387(10020):760-769.

33. Slavin MA, Thursky KA. Isavuconazole: a role for the newest broadspectrum triazole. Lancet. 2016;387(10020):726-728.

34. Park WB, Kim NH, Kim KH, et al. The effect of therapeutic drug monitoring on safety and efficacy of voriconazole in invasive fungal infections: a randomized controlled trial. Clin Infect Dis. 2012;55(8): 1080-1087.

35. Marty FM, Ostrosky-Zeichner L, Cornely OA, et al. Isavuconazole treatment for mucormycosis: a single-arm open-label trial and casecontrol analysis. Lancet Infect Dis. Epub 2016 Mar 8.

36. Kara IO, Tasova Y, Uguz A, Sahin B. Mucormycosis-associated fungal infections in patients with haematologic malignancies. Int J Clin Pract. 2009;63(1):134-139.

37. Greenberg RN, Mullane K, van Burik JA, et al. Posaconazole as salvage therapy for zygomycosis. Antimicrob Agents Chemother. 2006;50(1): 126-133.

38. van Burik JA, Hare RS, Solomon HF, Corrado ML, Kontoyiannis DP. Posaconazole is effective as salvage therapy in zygomycosis: a retrospective summary of 91 cases. Clin Infect Dis. 2006;42(7):e61-e65.

39. Falci DR, Pasqualotto AC. Profile of isavuconazole and its potential in the treatment of severe invasive fungal infections. Infect Drug Resist. 2013;6:163-174.
40. Pettit NN, Carver PL. Isavuconazole: A New Option for the Management of Invasive Fungal Infections. Ann Pharmacother. 2015;49(7): 825-842.

41. Shah RR. Drug-induced QT interval shortening: potential harbinger of proarrhythmia and regulatory perspectives. Br J Pharmacol. 2010; 159(1):58-69.

42. Shah RR, Bjerregaard P, Gussak I. Drug-induced QT interval shortening: an emerging component in integrated assessment of cardiac safety of drugs. J Electrocardiol. 2010;43(5):386-389.

43. Illnait-Zaragozi MT, Martinez GF, Curfs-Breuker I, Fernandez CM, Boekhout T, Meis JF. In Vitro activity of the new azole isavuconazole (BAL4815) compared with six other antifungal agents against 162 Cryptococcus neoformans isolates from Cuba. Antimicrob Agents Chemother. 2008;52(4):1580-1582.

44. Kim T, Jancel T, Kumar P, Freeman AF. Drug-drug interaction between isavuconazole and tacrolimus: a case report indicating the need for tacrolimus drug-level monitoring. J Clin Pharm Ther. Epub 2015 Aug 6.

45. Ashbee HR, Barnes RA, Johnson EM, Richardson MD, Gorton R, Hope WW. Therapeutic drug monitoring (TDM) of antifungal agents: guidelines from the British Society for Medical Mycology. J Antimicrob Chemother. 2014;69(5):1162-1176.

46. Ervens J, Ghannoum M, Graf B, Schwartz S. Successful isavuconazole salvage therapy in a patient with invasive mucormycosis. Infection. 2014;42(2):429-432.

47. Peixoto D, Gagne LS, Hammond SP, et al. Isavuconazole treatment of a patient with disseminated mucormycosis. J Clin Microbiol. 2014; 52(3):1016-1019.

48. Diflucan ${ }^{\circledR}$ (fluconazole) [package insert]. New York, NY: Pfizer; 2014.

49. Vfend ${ }^{\circledR}$ (voriconazole) [package insert]. New York, NY: Pfizer; 2015.

50. Seyedmousavi S, Verweij PE, Mouton JW. Isavuconazole, a broadspectrum triazole for the treatment of systemic fungal diseases. Expert Rev Anti Infect Ther. 2015;13(1):9-27.

51. Noxafil ${ }^{\circledR}$ (posaconazole) [package insert]. Whitehouse Station, NJ: Merck \& Co., Inc.; 2014.

52. Niwa T, Shiraga T, Takagi A. Effect of antifungal drugs on cytochrome P450 (CYP) 2C9, CYP2C19, and CYP3A4 activities in human liver microsomes. Biol Pharm Bull. 2005;28(9):1805-1808.

53. Bruggemann RJ, Alffenaar JW, Blijlevens NM, et al. Clinical relevance of the pharmacokinetic interactions of azole antifungal drugs with other coadministered agents. Clin Infect Dis. 2009;48(10):1441-1458.
Therapeutics and Clinical Risk Management

\section{Publish your work in this journal}

Therapeutics and Clinical Risk Management is an international, peerreviewed journal of clinical therapeutics and risk management, focusing on concise rapid reporting of clinical studies in all therapeutic areas, outcomes, safety, and programs for the effective, safe, and sustained use of medicines. This journal is indexed on PubMed Central, CAS,
Dovepress

EMBase, Scopus and the Elsevier Bibliographic databases. The manuscript management system is completely online and includes a very quick and fair peer-review system, which is all easy to use. Visit http://www.dovepress.com/testimonials.php to read real quotes from published authors. 